\title{
Association of dietary intakes of vitamin B12, vitamin B6, folate, and methionine with the risk of esophageal cancer: the Japan Public Health Center-based (JPHC) prospective study
}

Paramita Khairan ${ }^{1,2}$, Tomotaka Sobue ${ }^{1 *}$, Ehab Salah Eshak ${ }^{3,4}$, Ling Zha ${ }^{1}$, Tetsuhisa Kitamura ${ }^{1}$, Norie Sawada ${ }^{5}$, Motoki Iwasaki ${ }^{5}$, Manami Inoue ${ }^{5}$, Taiki Yamaji ${ }^{5}$, Taichi Shimazu ${ }^{5}$, Hiroyasu Iso ${ }^{3}$ and Shoichiro Tsugane ${ }^{5}$

\begin{abstract}
Background: B vitamins and methionine are essential substrates in the one-carbon metabolism pathway involved in DNA synthesis and methylation. They may have essential roles in cancer development. We aimed to evaluate the associations of dietary intakes of vitamin B12, vitamin B6, folate, and methionine with the risk of esophageal cancer (EC) using data from the Japan Public Health Center-based Prospective Study.

Methods: We included 87,053 Japanese individuals who completed a food frequency questionnaire and were followed up from 1995-1998 to 2013 and 2015. Hazard ratios (HRs) and 95\% confidence intervals (Cls) were calculated by Cox proportional-hazard regression across quintiles of dietary intakes of B vitamins and methionine.

Results: After 1,456,678 person-years of follow-up, 427 EC cases were documented. The multivariable HR (95\% Cl) of incident EC in the highest versus lowest quintile of dietary intake of vitamin B12 was 1.75 (1.13-2.71; $p$-trend= 0.01). Stratification analysis based on alcohol consumption showed that higher dietary intakes of vitamin B12 and methionine were associated with an increased risk of EC among never-drinkers; HRs (95\% Cls) were 2.82 (1.18-6.74; $p$-trend=0.009; $p$-interaction=0.18) and 3.45 (1.32-9.06; $p$-trend=0.003; $p$-interaction 0.02) for vitamin B12 and methionine, respectively. Meanwhile, there was no association between vitamin B12 and methionine intake with the risk of $\mathrm{EC}$ among drinkers. There were no associations between dietary intake of folate or vitamin $\mathrm{B} 6$ and the risk of EC.
\end{abstract}

Conclusion: Dietary intake of vitamin B12 was positively associated with the risk of EC in the Japanese population. Keywords: Esophageal cancer, One-carbon metabolism, Vitamin B12 intake, Folate intake, Prospective cohort study

\footnotetext{
* Correspondence: tsobue@envi.med.osaka-u.ac.jp

'Department of Social and Environmental Medicine, Graduate School of Medicine, Osaka University, 2-2 Yamadaoka, Suita, Osaka 565-0871, Japan Full list of author information is available at the end of the article
}

C C The Author(s). 2021 Open Access This article is licensed under a Creative Commons Attribution 4.0 International License, which permits use, sharing, adaptation, distribution and reproduction in any medium or format, as long as you give appropriate credit to the original author(s) and the source, provide a link to the Creative Commons licence, and indicate if changes were made. The images or other third party material in this article are included in the article's Creative Commons. licence, unless indicated otherwise in a credit line to the material. If material is not included in the article's Creative Commons licence and your intended use is not permitted by statutory regulation or exceeds the permitted use, you will need to obtain permission directly from the copyright holder. To view a copy of this licence, visit http://creativecommons.org/licenses/by/4.0/ The Creative Commons Public Domain Dedication waiver (http://creativecommons.org/publicdomain/zero/1.0/) applies to the data made available in this article, unless otherwise stated in a credit line to the data. 


\section{Background}

Esophageal cancer (EC) is the sixth most common cause of cancer-related mortality worldwide, particularly in eastern Asian and eastern African regions [1]. Esophageal squamous cell carcinomas account for $88 \%$ of EC cases, with an increasing trend of incidence among women in several countries, including Japan [2].

Lifestyle habits such as alcohol consumption and tobacco smoking are the main risk factors for esophageal squamous cell carcinoma [3]. It has been suggested that diets rich in foods of animal origin and low in foods containing vitamins $\mathrm{C}$ and $\mathrm{E}$ are associated with an increased risk of EC [4]. Specifically, the total intake of red meat (supposedly high in vitamin B12) has been positively associated with an increased risk of EC $[5,6]$.

Vitamin B12 and folate are the primary components of the one-carbon metabolism (OCM) pathway involved in DNA synthesis, repair, and methylation [7, 8]. Additionally, vitamin B6 and methionine are also co-factors that are required in the OCM pathway [9]. A dietary imbalance or deficiency in those nutrients may disrupt DNA methylation or induce the disincorporation of nucleotide synthesis, which could lead to carcinogenesis [9].

Recently, the dietary intakes of folate and vitamin B12 were shown to be associated with the risk of several cancers, such as colorectal, breast, pancreatic, and lung cancers [10-13]. The associations of folate and vitamin B12 intakes with EC risk have been inconsistent, and most of the studies that investigated these associations did not utilize a prospective design [14-19]. Two previous case-control studies that investigated the association between EC risk and dietary folate intake reported reduced EC risk, even among smokers and alcohol drinkers $[15,16]$. Alcohol drinking, a strong risk factor for EC, may modify the association between dietary intakes of $\mathrm{B}$ vitamins and the risk of EC because alcohol affects the absorption and metabolism of vitamin B12 and folate [20, 21].

Herein, we conducted a prospective cohort study in a large Japanese population to clarify the association of dietary intake of vitamin B12, vitamin B6, folate, and methionine with the risk of $\mathrm{EC}$ and to investigate the hypothesized potential effect modifications by alcohol consumption.

\section{Methods}

\section{Study design and participants}

The Japan Public Health Center-based Prospective Study (JPHC Study) is a large population-based cohort study that began in 1990-1994 to investigate the risk factors for cancer, metabolic diseases, and other lifestyle-related diseases. Complete details of the study design are available elsewhere [22]. This study was approved by the Institutional Review Board of the National Cancer Center, Japan, and Osaka University.
The participants eligible for this study were those who participated in the JPHC study who gave feedback in the 5-year follow-up self-administered questionnaire surveys. The 5-year follow-up self-administered questionnaire surveys was including more comprehensive information of food intake's frequency. The surveys were distributed to residents of 11 public health center areas in 1995-1999 who were aged 45-74 years. Participants registered in Tokyo and Suita areas were excluded because of information's unavailability on cancer incidence in Tokyo area and a different study populations' definition in Suita area. We excluded participants who met one or more of the following exclusion criteria: diagnosis of EC during the period between the baseline survey administration and 5-year follow-up survey $(n=18)$, history of cancer $(n=1,250)$, missing values for the nutrients evaluated in the study $(n=1,068)$, or excessive energy intake (subjects who reported energy intake in the upper or lower $1 \%$ of intake $[n=1,774])$. Finally, data of a total of 87,053 men and women were eligible for the analysis. Details are shown in the flow chart of the study participants (Fig. 1).

\section{Assessment of exposures and covariates}

The 5-year follow-up questionnaire survey included a food frequency questionnaire (FFQ) to estimate the dietary intake of 147 food items. Responses of diet frequency for each food item ranged from "rarely," "1-3 days/ month," "1-2 days/week," "3-4 days/week," or "5-6 days/week," to "once/day," "2-3 times/day," "4-6 times/ day," or " $\geq 7$ times/day." Energy-adjusted dietary intakes of vitamin B12, vitamin B6, folate, and methionine were calculated using the residual method, and daily nutrient intake was calculated using the Standard Table of Food consumption in Japan, $7^{\text {th }}$ revised and enlarged edition $[23,24]$. The supplemental nutrition intake was not included in the current analysis because of the lack of a database of the supplements. The validity of vitamin $\mathrm{B} 12$, vitamin $\mathrm{B} 6$, folate, and methionine estimated from the FFQ was evaluated from a subsample of participants in the JPHC Study Cohorts I and II $[25,26]$. Regarding the validity of the FFQ, energy-adjusted Spearman's correlation coefficients between intake values of vitamin $\mathrm{B} 12$, vitamin $\mathrm{B} 6$, and folate derived from the FFQ and those derived from the 28-day or 14-day dietary records in men were $0.33,0.45$, and 0.40 in Cohort I, respectively, and 0.35, 0.36, 0.50 in Cohort II, respectively. For women, the correlation coefficients were $0.34,0.47$, and 0.35 in Cohort I, respectively, and 0.27, 0.40, and 0.48 in Cohort II, respectively [26]. Regarding the validity of the FFQ for methionine intake, Spearman's correlation coefficients were 0.29 and 0.27 for men and women, respectively, in Cohort I. In Cohort II, the values were 0.27 and 0.31 for men and women, respectively [25]. 


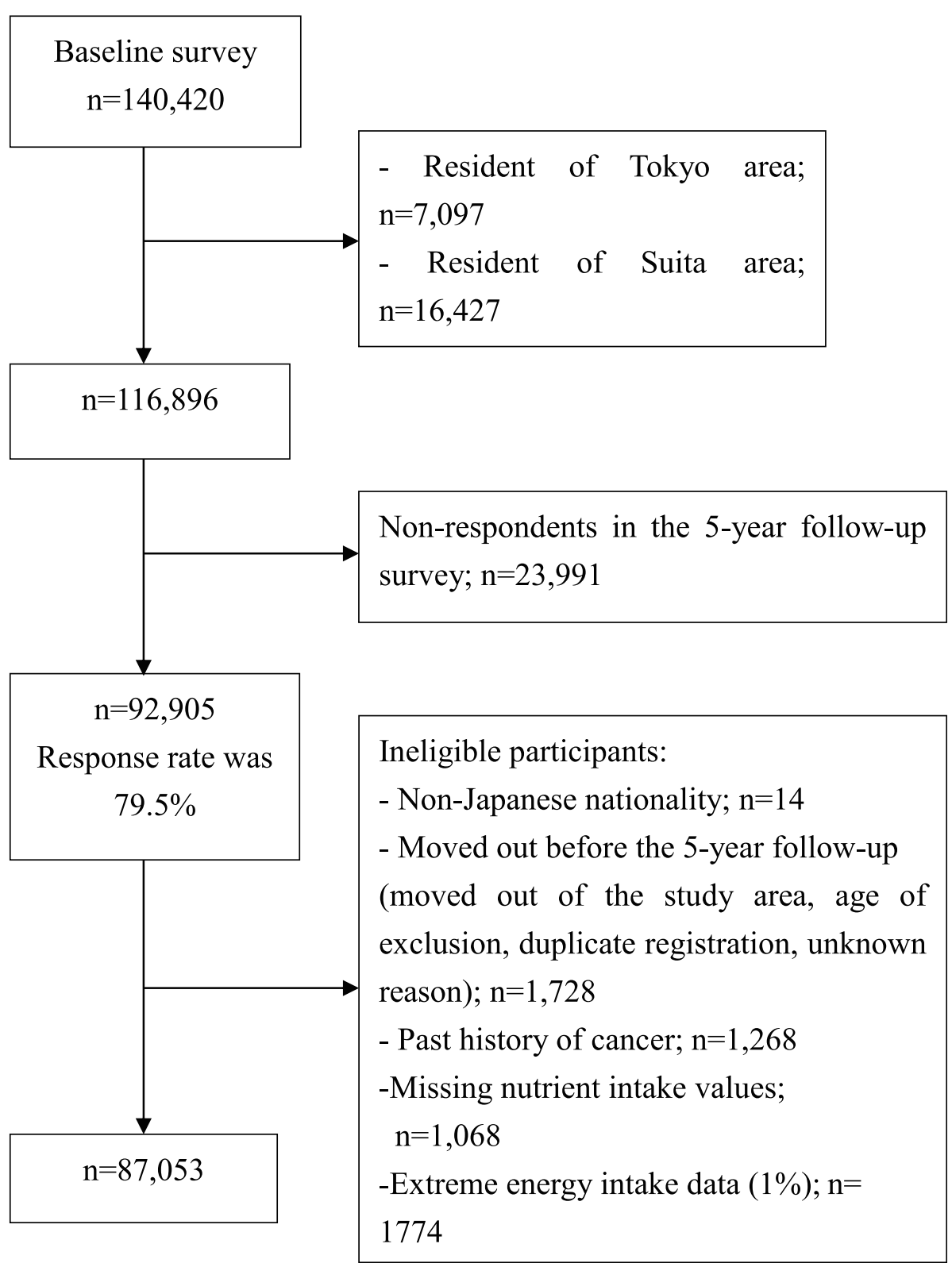

Fig. 1 Flow chart of the study participants involved in the 5-year follow-up survey (baseline of the current study)

The questionnaire also asked the participants to report their demographic characteristics, medical history, smoking and drinking habits, lifestyle, occupation, height and weight, physical activity, working hours, stress, and other life habits.

\section{Ascertainment of EC cases}

EC data were collected from the cancer registries, medical records from hospitals in the study areas, reviews of the death certificate, or both medical records and death certificates. We identified the cases using the International Classification of Disease for Oncology Codes, third edition code "C15" with histology code 8050-8084 [27].

\section{Statistical analyses}

Statistical analyses were based on EC incidence values during 15.8 median years of follow-up until December 31, 2013 for Kochi and Nagasaki area, and December 31, 2015 for the other PHC areas. For each individual, person-years of follow-up were calculated from the day of submitting the 5-year follow-up survey to whichever of these endpoints occurred first: diagnosis of EC, death, emigration, or December 31, 2013. The hazard ratios (HRs) with 95\% confidence intervals (CIs) of the incidence of EC were calculated using time-dependent Cox proportional hazard models according to the quintiles of dietary intakes of vitamin 
B12, vitamin B6, folate, and methionine after adjusting for age (5-year categories), sex, and study area in Model 1. Model 2 was additionally adjusted for quintiles of the body mass index in $\mathrm{kg} / \mathrm{m}^{2}$, physical activity in metabolic equivalent of task unit (quintiles), alcohol consumption (not current drinker, current drinker of $\leq 150 \mathrm{~g} /$ week, $151-\leq 300 \mathrm{~g} /$ week, 301-<450 g/week, $\geq 450 \mathrm{~g} /$ week, or missing), smoking (never, former, current smoker of $\leq 19$ cigarettes/day, 20-29 cigarettes/day, $\geq 30$ cigarettes/day, or missing), and family history of cancer (yes or no). Model 3 was adjusted further for vitamin B12, vitamin B6, folate, and methionine mutually to estimate the independent effect of the nutrients. Sensitivity analysis was conducted by excluding EC cases diagnosed within three years after the study's enrolment. We also performed tests for linear trends across the intake quintile categories of the studied $B$ vitamins and methionine by adding to the model and testing the significance of variables representing each category's median values.

As alcohol is a strong risk factor for EC and affects the absorption and metabolism of B vitamins [20, 21], and as Japanese men are more likely to drink than women [28], we conducted stratified analyses based on alcohol intake status (never-drinker, low-drinker/alcohol intake 0 -150 g/week, and high-drinker/alcohol intake> $150 \mathrm{~g} /$ week) in all participants and men. We attained the $p$-trend for each level of alcohol intake and $p$-interaction for a cross-product term of the alcohol categories and rank variables for quintiles of dietary intakes of $\mathrm{B}$ vitamins and methionine. All statistical analyses were two-tailed, with $p<0.05$ as the statistically significant level, and were conducted using SAS software (version 9.4; SAS Institute).

\section{Results}

Table 1 shows the distribution of EC risk factors across the quintiles of dietary intake of vitamin B12, vitamin B6, folate, and methionine. The participants in the highest quintile of dietary intakes of these nutrients tended to be slightly older, consumed less alcohol, and were less likely to smoke (except for the high smoking prevalence in the highest quintile of dietary vitamin B12 intake).

During 1,456, 678 person-years of follow-up of 87,053 Japanese men and women aged 45-74 years, we documented 427 incident cases of EC (382 men and 45 women) comprising 332 esophageal squamous cell carcinomas, 17 esophageal adenocarcinomas, and 78 non-specific EC.

Table 2 presents the HRs of EC according to dietary intake of vitamin B12, vitamin B6, folate, and methionine. The dietary intake of vitamin B12 showed a positive association with the risk of EC (Model 2; HR and 95\% CI in the highest versus lowest quintiles was 1.37
[1.00-1.86]; $p$-trend=0.04) (Table 2). The positive association between vitamin B12 intake and risk of EC became stronger after further adjustment for vitamin B6, folate, and methionine (Model 3; HR and 95\% CI: 1.75 [1.13$2.71] ; p$-trend $=0.01)$. After excluding EC cases diagnosed within three years of enrolment, the association did not alter materially (Model 3: $\mathrm{HR}$ and 95\% CI: 1.74[1.09-2.78]; p-trend $=0.02$ ). No associations were observed between the dietary intakes of vitamin B6, folate, and methionine and risk of EC (Table 2).

In the associations of the dietary intakes of both vitamin B12 and methionine with the risk of EC, alcohol intake confounded the associations. The adjustment for alcohol intake contributed to the increase in EC risk estimates across the dietary intake quintiles of vitamin B12 and disappearance of the inverse association observed for folate and methionine. As alcohol consumption differs greatly according to sex, we tested the sex-specific associations of dietary intakes of vitamins B12, folate, and methionine with the risk of EC and found no significant sex-specific interactions $(p>0.1)$. The sex-specific associations did not reach a level of significance in either sex due to the loss of power (data not shown in table).

We conducted stratified analyses based on alcohol intake status for all participants (Table 3) and for men (Supplementary Table 1). The significant positive association between dietary intake of vitamin B12 and the risk of EC was only evident for participants who never consumed alcohol; however, the p-interaction did not reach the level of significance $(p=0.18)$. A similar positive association was found for higher methionine intake in never drinkers, and the interaction with alcohol reached the level of statistical significance $(p=0.02)$. The multivariable HRs (95\% CIs) in the highest versus lowest quintiles of dietary intakes of vitamin B12 and methionine among alcohol never-drinkers were 2.82 (1.18-6.74; $p$-trend= $0.009)$ and 3.45 (1.32-9.06; $p$-trend $=0.003)$, respectively (Table 3). The HRs (95\% CIs) in the highest versus lowest tertiles of dietary intakes of vitamin B12 and methionine among men who were never-drinkers were 2.92 $(0.93-9.19 ; p$-trend $=0.02 ; p$-interaction $=0.24)$ and 3.30 $(0.77-14.10 ; p$-trend $=0.004 ; p$-interaction $=0.03)$, respectively (Supplementary Table 1). There were no interactions by alcohol intake on the association of dietary intakes of vitamin B6 and folate with the risk of EC (Table 3).

\section{Discussion}

In this large prospective study of Japanese men and women, we found that a higher dietary intake of vitamin B12 was associated with an increased risk of EC after adjusting for EC risk factors. When stratified by alcohol drinking status, we found positive associations of vitamin $\mathrm{B} 12$ and methionine with the risk of EC among never- 
Table 1 Baseline characteristics of the participants according to quintiles of energy-adjusted dietary intakes of vitamin B12, vitamin B6, folate, and methionine

\begin{tabular}{|c|c|c|c|c|c|c|}
\hline Characteristics & Q1 & Q2 & Q3 & Q4 & Q5 & $p$ value $^{\mathrm{a}}$ \\
\hline \multicolumn{7}{|l|}{ Vitamin B12 $^{\mathrm{b}}$} \\
\hline No. of participants & 17,410 & 17,411 & 17,411 & 17,411 & 17,410 & \\
\hline Male, n (\%) & $8,003(46.0)$ & $7,555(43.4)$ & $7,978(45.8)$ & $8,290(47.6)$ & $8,890(51.1)$ & $<.0001$ \\
\hline Age, years ${ }^{c}$ & $56.2 \pm 7.9$ & $56.7 \pm 8.0$ & $56.8 \pm 8.0$ & $57.2 \pm 7.7$ & $57.7 \pm 7.5$ & $<.0001$ \\
\hline $\mathrm{BMI}, \mathrm{kg} / \mathrm{m}^{2 \mathrm{c}}$ & $23.8 \pm 3.5$ & $23.7 \pm 3.5$ & $23.5 \pm 3.2$ & $23.5 \pm 3.3$ & $23.5 \pm 3.4$ & $<.0001$ \\
\hline Current smokers, n (\%) & $3,877(22.3)$ & $3,672(21.1)$ & $3,783(21.7)$ & $3,927(22.6)$ & $4,396(25.3)$ & $<.0001$ \\
\hline Family history of cancer, yes ( $\mathrm{n}[\%]$ ) & 2,249 (12.9) & $2,454(14.1)$ & $2,627(15.1)$ & $2,829(16.3)$ & $2,828(16.2)$ & $<.0001$ \\
\hline METs unit ${ }^{c}$ & $34.2 \pm 6.3$ & $33.5 \pm 6.0$ & $33.2 \pm 5.9$ & $33.3 \pm 6.0$ & $33.6 \pm 6.1$ & $<.0001$ \\
\hline Alcohol consumption, ethanol g/week ${ }^{c}$ & $163.9 \pm 299.4$ & $94.8 \pm 180.3$ & $84.6 \pm 163.8$ & $85.8 \pm 162.5$ & $102.7 \pm 180.7$ & $<.0001$ \\
\hline Alcohol, n (\%) & & & & & & 0.004 \\
\hline Non-drinkers & $9,333(53.6)$ & $9,956(57.2)$ & $9,899(56.9)$ & $9,591(55.1)$ & $9,224(53.0)$ & \\
\hline$\leq 150 \mathrm{~g} /$ week & $2,554(14.7)$ & $3,139(18.0)$ & 3,425 (19.7) & $3,695(21.2)$ & $3,380(19.4)$ & \\
\hline $150<$ alcohol $\leq 300 \mathrm{~g} /$ week & $1,282(7.4)$ & $1,497(8.6)$ & $1,646(9.5)$ & $1,672(9.6)$ & $1,827(10.5)$ & \\
\hline $300<$ alcohol<450 g/week & $1,260(7.2)$ & $1,158(6.7)$ & $1,107(6.4)$ & $1,134(6.5)$ & $1,302(7.5)$ & \\
\hline$\geq 450 \mathrm{~g} /$ week & $2,619(15.0)$ & $1,226(7.0)$ & $912(5.2)$ & $917(5.3)$ & $1,243(7.1)$ & \\
\hline Energy, kcal/day ${ }^{c}$ & $2,398.1 \pm 710.5$ & $1,859.0 \pm 572.3$ & $1,774.0 \pm 602.0$ & $1,854.9 \pm 627.3$ & $2,218.3 \pm 746.4$ & $<.0001$ \\
\hline Vitamin B6, mg/day ${ }^{c}$ & $1.3 \pm 0.4$ & $1.4 \pm 0.2$ & $1.5 \pm 0.2$ & $1.6 \pm 0.2$ & $1.9 \pm 0.4$ & $<.0001$ \\
\hline Vitamin B12, $\mu \mathrm{g} /$ day $^{\mathrm{C}}$ & $3.7 \pm 2.6$ & $7.4 \pm 0.6$ & $9.3 \pm 0.6$ & $11.6 \pm 0.8$ & $18.3 \pm 6.7$ & $<.0001$ \\
\hline Folate, $\mu \mathrm{g} /$ day $^{c}$ & $361.8 \pm 200.0$ & $392.5 \pm 135.3$ & $406.5 \pm 127.7$ & $425.6 \pm 132.1$ & $453.7 \pm 166.4$ & $<.0001$ \\
\hline Methionine, mg/day ${ }^{c}$ & $1,293.0 \pm 363.3$ & $1,525.3 \pm 215.1$ & $1,638.0 \pm 211.3$ & $1,763.6 \pm 230.0$ & $2,079.1 \pm 469.0$ & $<.0001$ \\
\hline \multicolumn{7}{|l|}{ Vitamin B6 ${ }^{\text {b }}$} \\
\hline No. of participants & 17,410 & 17,411 & 17,411 & 17,411 & 17,410 & \\
\hline Male, n (\%) & $6,848(39.3)$ & $6,429(36.9)$ & $7,768(44.6)$ & $9,091(52.2)$ & $10,580(60.8)$ & $<.0001$ \\
\hline Age, years ${ }^{c}$ & $55.0 \pm 7.6$ & $56.3 \pm 7.9$ & $57.2 \pm 7.9$ & $57.7 \pm 7.7$ & $58.5 \pm 7.6$ & $<.0001$ \\
\hline $\mathrm{BMI}, \mathrm{kg} / \mathrm{m}^{2 \mathrm{C}}$ & $23.7 \pm 3.4$ & $23.5 \pm 3.3$ & $23.5 \pm 3.3$ & $23.6 \pm 3.5$ & $23.7 \pm 3.4$ & 0.05 \\
\hline Current smokers, n (\%) & $4,237(24.3)$ & $3,511(20.2)$ & $3,654(21.0)$ & $3,986(22.9)$ & $4,268(24.5)$ & $<.0001$ \\
\hline Family history of cancer, yes ( $\mathrm{n}[\%]$ ) & 2,191 (12.6) & $2,423(13.9)$ & $2,673(15.4)$ & $2,744(15.8)$ & $2,956(17.0)$ & $<.0001$ \\
\hline METs unit ${ }^{c}$ & $33.8 \pm 6.2$ & $33.2 \pm 5.9$ & $33.4 \pm 6.0$ & $33.5 \pm 6.1$ & $33.8 \pm 6.2$ & $<.0001$ \\
\hline Alcohol consumption, ethanol g/week ${ }^{c}$ & $151.1 \pm 296.8$ & $80.5 \pm 172.6$ & $80.8 \pm 163.6$ & $95.1 \pm 167.8$ & $124.1 \pm 188.7$ & $<.0001$ \\
\hline Alcohol, n (\%) & & & & & & $<.0001$ \\
\hline Non-drinkers & $9,988(57.4)$ & $1,0644(61.1)$ & $10,082(57.9)$ & $9,151(52.6)$ & $8,135(46.7)$ & \\
\hline$\leq 150 \mathrm{~g} /$ week & $2,627(15.1)$ & $3,104(17.8)$ & $3,494(20.1)$ & $3,621(20.8)$ & $3,347(19.2)$ & \\
\hline $150<$ alcohol $\leq 300 \mathrm{~g} /$ week & $1,060(6.1)$ & $1,269(7.3)$ & $1,465(8.4)$ & $1,916(11.0)$ & $2,214(12.7)$ & \\
\hline $300<$ alcohol<450 g/week & $824(4.7)$ & $912(5.2)$ & $1,043(6.0)$ & $1,338(7.7)$ & $1,845(10.6)$ & \\
\hline$\geq 450 \mathrm{~g} /$ week & $2,590(14.9)$ & $1,031(5.9)$ & $875(5.0)$ & $984(5.7)$ & $1,434(8.2)$ & \\
\hline Energy, kcal/day ${ }^{\mathrm{c}}$ & $2,265.4 \pm 735.6$ & $1,833.1 \pm 615.5$ & $1,817.3 \pm 609.6$ & $1,917.5 \pm 623.3$ & $2,270.8 \pm 742.7$ & $<.0001$ \\
\hline Vitamin B6, mg/day ${ }^{c}$ & $1.1 \pm 0.2$ & $1.4 \pm 0.04$ & $1.5 \pm 0.04$ & $1.7 \pm 0.1$ & $2.1 \pm 0.3$ & $<.0001$ \\
\hline Vitamin B12, $\mu \mathrm{g} /$ day $^{\mathrm{C}}$ & $5.7 \pm 4.0$ & $8.5 \pm 3.2$ & $9.7 \pm 3.5$ & $11.2 \pm 4.2$ & $15.0 \pm 8.2$ & $<.0001$ \\
\hline Folate, $\mu \mathrm{g} /$ day ${ }^{c}$ & $280.8 \pm 109.9$ & $364.3 \pm 94.4$ & $405.6 \pm 102.8$ & $449.7 \pm 121.3$ & $539.6 \pm 202.7$ & $<.0001$ \\
\hline Methionine, mg/day ${ }^{c}$ & $1,377.7 \pm 371.2$ & $1,561.9 \pm 242.1$ & $1,644.6 \pm 252.9$ & $1,736.3 \pm 286.3$ & $1,978.2 \pm 544.5$ & $<.0001$ \\
\hline \multicolumn{7}{|l|}{ Folate $^{b}$} \\
\hline No. of participants & 17,410 & 17,411 & 17,411 & 17,411 & 17,410 & $<.0001$ \\
\hline Male, n (\%) & $10,072(57.9)$ & $8,732(50.2)$ & $7,646(43.9)$ & $7,310(42.0)$ & $6,956(40.0)$ & $<.0001$ \\
\hline
\end{tabular}


Table 1 Baseline characteristics of the participants according to quintiles of energy-adjusted dietary intakes of vitamin B12, vitamin B6, folate, and methionine (Continued)

\begin{tabular}{|c|c|c|c|c|c|c|}
\hline Characteristics & Q1 & Q2 & Q3 & Q4 & Q5 & $p$ value $^{\mathrm{a}}$ \\
\hline Age, years ${ }^{c}$ & $54.9 \pm 7.5$ & $56.3 \pm 7.9$ & $57.0 \pm 7.9$ & $57.8 \pm 7.8$ & $58.7 \pm 7.7$ & $<.0001$ \\
\hline BMl, $\mathrm{kg} / \mathrm{m}^{2 \mathrm{c}}$ & $23.7 \pm 3.3$ & $23.6 \pm 3.4$ & $23.5 \pm 3.3$ & $23.6 \pm 3.4$ & $23.6 \pm 3.5$ & 0.03 \\
\hline Current smokers, n (\%) & $5,664(32.5)$ & $4,148(23.8)$ & $3,557(20.4)$ & $3,254(18.7)$ & $3,033(17.4)$ & $<.0001$ \\
\hline Family history of cancer, yes (n [\%]) & $2,222(12.8)$ & $2,343(13.5)$ & $2,650(15.2)$ & $2,814(16.2)$ & $2,958(17.0)$ & $<.0001$ \\
\hline METs unit ${ }^{c}$ & $33.9 \pm 6.4$ & $33.3 \pm 6.1$ & $33.3 \pm 6.0$ & $33.6 \pm 6.0$ & $33.7 \pm 6.0$ & 0.004 \\
\hline Alcohol consumption, ethanol $\mathrm{g} /$ week $^{c}$ & $209.3 \pm 311.1$ & $103.7 \pm 182.4$ & $82.0 \pm 159.2$ & $72.6 \pm 150.4$ & $63.4 \pm 140.6$ & $<.0001$ \\
\hline Alcohol intake, n (\%) & & & & & & $<.0001$ \\
\hline Non-drinkers & $7,560(43.4)$ & $9,272(53.3)$ & $9,943(57.1)$ & $10,401(59.7)$ & $10,819(62.1)$ & \\
\hline$\leq 150 \mathrm{~g} /$ week & $2,661(15.3)$ & 3,338 (19.2) & 3,452 (19.8) & 3,397 (19.8) & $3,345(19.2)$ & \\
\hline $150<$ alcohol $\leq 300 \mathrm{~g} /$ week & $1,695(9.7)$ & $1,797(10.3)$ & $1,600(9.2)$ & $1,490(8.6)$ & $1,342(7.7)$ & \\
\hline $300<$ alcohol<450 g/week & $1,701(9.8)$ & $1,346(7.7)$ & $1,087(6.2)$ & $956(5.5)$ & $871(5.0)$ & \\
\hline$\geq 450 \mathrm{~g} /$ week & $3,496(20.1)$ & $1,247(7.2)$ & $861(5.0)$ & $737(4.2)$ & $576(3.3)$ & \\
\hline Energy, kcal/day ${ }^{c}$ & $2,335.4 \pm 727.8$ & $1,880.2 \pm 634.5$ & $1,837.6 \pm 622.5$ & $1,910.2 \pm 645.3$ & $2,140.9 \pm 725.3$ & $<.0001$ \\
\hline Vitamin B6, mg/day ${ }^{c}$ & $1.3 \pm 0.4$ & $1.5 \pm 0.3$ & $1.6 \pm 0.3$ & $1.6 \pm 0.3$ & $1.8 \pm 0.4$ & $<.0001$ \\
\hline Vitamin B12, $\mu \mathrm{g} /$ day $^{c}$ & $8.0 \pm 6.7$ & $9.7 \pm 4.7$ & $10.4 \pm 4.9$ & $10.8 \pm 5.0$ & $11.4 \pm 6.9$ & $<.0001$ \\
\hline Folate, $\mu \mathrm{g} /$ day $^{c}$ & $224.5 \pm 68.2$ & $326.7 \pm 18.4$ & $388.1 \pm 18.0$ & $461.6 \pm 26.1$ & $639.2 \pm 146.7$ & $<.0001$ \\
\hline Methionine, mg/day ${ }^{c}$ & $1,547.7 \pm 525.4$ & $1,655.1 \pm 346.1$ & $1,691.4 \pm 342.1$ & $1,706.8 \pm 348.6$ & $1,698.1 \pm 428.7$ & $<.0001$ \\
\hline \multicolumn{7}{|l|}{ Methionine ${ }^{b}$} \\
\hline No. of participants & 17,410 & 17,411 & 17,411 & 17,411 & 17,410 & \\
\hline Male, n (\%) & $7,924(45.5)$ & $6,273(36.0)$ & $7,211(41.4)$ & $8,748(50.2)$ & $10,560(60.7)$ & $<.0001$ \\
\hline Age, years ${ }^{c}$ & $55.7 \pm 7.8$ & $56.7 \pm 7.9$ & $57.1 \pm 8.0$ & $57.3 \pm 7.8$ & $57.8 \pm 7.6$ & $<.0001$ \\
\hline $\mathrm{BMI}, \mathrm{kg} / \mathrm{m}^{2 \mathrm{c}}$ & $23.6 \pm 3.4$ & $23.6 \pm 3.5$ & $23.6 \pm 3.4$ & $23.6 \pm 3.3$ & $23.6 \pm 3.3$ & 0.06 \\
\hline Current-smokers, n (\%) & $4,620(26.5)$ & 3,364 (19.3) & $3,439(19.8)$ & 3,811 (21.9) & $4,422(25.4)$ & $<.0001$ \\
\hline Family history of cancer, yes ( $\mathrm{n}[\%]$ ) & $2,460(14.1)$ & $2,519(14.5)$ & $2,667(15.3)$ & $2,649(15.2)$ & $2,692(15.5)$ & 0.006 \\
\hline METs unit ${ }^{c}$ & $34.2 \pm 6.3$ & $33.5 \pm 6.0$ & $33.3 \pm 5.9$ & $33.2 \pm 6.0$ & $33.4 \pm 6.1$ & $<.0001$ \\
\hline Alcohol intake, ethanol g/week ${ }^{c}$ & $221.4 \pm 329.4$ & $90.4 \pm 167.2$ & $71.8 \pm 139.4$ & $67.0 \pm 131.4$ & $80.8 \pm 148.2$ & $<.0001$ \\
\hline Alcohol intake, n (\%) & & & & & & $<.0001$ \\
\hline Non-drinkers & $8,214(47.2)$ & $10,251(58.9)$ & $10,241(58.8)$ & $9,929(57.0)$ & $9,368(53.8)$ & \\
\hline$\leq 150 \mathrm{~g} /$ week & $2,031(11.7)$ & $2,743(15.8)$ & 3,445 (19.8) & $4,001(23.0)$ & $3,978(22.9)$ & \\
\hline $150<$ alcohol $\leq 300 \mathrm{~g} /$ week & $1,249(7.2)$ & $1,532(8.8)$ & $1,635(9.4)$ & $1,665(9.6)$ & $1,843(10.6)$ & \\
\hline $300<$ alcohol<450 g/week & $1,692(9.7)$ & $1,326(7.6)$ & $1,023(5.9)$ & $881(5.1)$ & $1,039(6.0)$ & \\
\hline$\geq 450 \mathrm{~g} /$ week & $3,911(22.5)$ & $1,100(6.3)$ & $654(3.8)$ & $521(3.0)$ & $731(4.2)$ & \\
\hline Energy, kcal/day ${ }^{c}$ & $2,323.2 \pm 701.9$ & $1,859.0 \pm 594.3$ & $1,799.7 \pm 602.0$ & $1,866.1 \pm 622.7$ & $2,256.3 \pm 774.2$ & $<.0001$ \\
\hline Vitamin B6, mg/day ${ }^{c}$ & $1.3 \pm 0.4$ & $1.5 \pm 0.2$ & $1.5 \pm 0.2$ & $1.6 \pm 0.2$ & $1.9 \pm 0.4$ & $<.0001$ \\
\hline Vitamin B12, $\mu \mathrm{g} /$ day $^{\mathrm{C}}$ & $5.0 \pm 3.7$ & $8.0 \pm 2.7$ & $9.6 \pm 2.8$ & $11.4 \pm 3.5$ & $16.2 \pm 7.9$ & $<.0001$ \\
\hline Folate, $\mu \mathrm{g} /$ day ${ }^{c}$ & $378.4 \pm 202.1$ & $401.1 \pm 141.2$ & $410.2 \pm 130.6$ & $420.0 \pm 134.4$ & $430.4 \pm 164.4$ & $<.0001$ \\
\hline Methionine, mg/day ${ }^{c}$ & $1,169.7 \pm 247.7$ & $1,485.1 \pm 47.3$ & $1,633.3 \pm 41.6$ & $1,797.1 \pm 56.3$ & $2,213.9 \pm 411.9$ & $<.0001$ \\
\hline
\end{tabular}

${ }^{a}$ Chi-square test for qualitative variables, ANOVA for continuous variables.

${ }^{b}$ Cut-offs for quintiles of dietary intake of vitamin B12 were $6.24,8.35,10.28$, and $13.2 \mu \mathrm{g} /$ day. Cut-offs for quintiles of dietary intake of vitamin B6 were $1.31,1.46$, 1.61 , and $1.79 \mathrm{mg} /$ day. Cut-offs for quintiles of dietary intake of folate were $293.24,357.78,420.28$, and $511.80 \mu \mathrm{g} /$ day. Cut-offs for quintiles of dietary intake of methionine were $1395.84,1562.41,1707.36$, and $1904.13 \mathrm{mg} /$ day.

c Mean \pm standard deviation, all such variables 
Table 2 Hazard ratios (95\% confident intervals) of esophageal cancer according to quintiles of energy-adjusted dietary intakes of vitamin B12, vitamin B6, folate, and methionine

\begin{tabular}{|c|c|c|c|c|c|c|}
\hline & Q1 & Q2 & Q3 & Q4 & Q5 & $p$-trend $^{a}$ \\
\hline \multicolumn{7}{|l|}{ Vitamin B12 } \\
\hline Person-years & 296,280 & 292,419 & 290,662 & 289,062 & 288,256 & \\
\hline Cases, n & 80 & 72 & 82 & 87 & 106 & \\
\hline Model $1^{\mathrm{b}}$ & 1.00 (reference) & $0.93(0.68-1.28)$ & $1.00(0.73-1.37)$ & $1.00(0.73-1.37)$ & $1.13(0.83-1.52)$ & 0.31 \\
\hline Model $2^{c}$ & 1.00 (reference) & $1.09(0.79-1.51)$ & $1.25(0.91-1.72)$ & $1.28(0.93-1.76)$ & $1.37(1.00-1.86)$ & 0.04 \\
\hline Model $3^{d}$ & 1.00 (reference) & $1.13(0.80-1.59)$ & $1.43(0.99-2.06)$ & $1.58(1.07-2.34)$ & $1.75(1.13-2.71)$ & 0.01 \\
\hline \multicolumn{7}{|c|}{ 3-year exclusion analysis } \\
\hline Cases, n & 71 & 64 & 71 & 79 & 91 & \\
\hline Model $3^{d}$ & 1.00 (reference) & $1.13(0.79-1.63)$ & $1.40(0.95-2.06)$ & $1.64(1.08-2.48)$ & $1.74(1.09-2.78)$ & 0.02 \\
\hline \multicolumn{7}{|l|}{ Vitamin B6 } \\
\hline Person-years & 296,267 & 294,533 & 291,293 & 288,886 & 285,698 & \\
\hline Cases, n & 75 & 78 & 82 & 86 & 106 & \\
\hline Model $1^{\mathrm{b}}$ & 1.00 (reference) & $1.01(0.73-1.39)$ & $0.87(0.63-1.19)$ & $0.78(0.57-1.07)$ & $0.81(0.60-1.09)$ & 0.08 \\
\hline Model $2^{c}$ & 1.00 (reference) & $1.24(0.90-1.71)$ & $1.16(0.84-1.60)$ & $1.08(0.78-1.49)$ & $1.08(0.79-1.48)$ & 0.98 \\
\hline Model $3^{d}$ & 1.00 (reference) & $1.17(0.83-1.64)$ & $1.04(0.72-1.48)$ & $0.89(0.61-1.30)$ & $0.81(0.53-1.24)$ & 0.16 \\
\hline \multicolumn{7}{|c|}{ 3-year exclusion analysis } \\
\hline Cases, n & 65 & 70 & 73 & 75 & 93 & \\
\hline Model $3^{d}$ & 1.00 (reference) & $1.22(0.85-1.76)$ & $1.09(0.74-1.60)$ & $0.94(0.63-1.42)$ & $0.89(0.57-1.40)$ & 0.36 \\
\hline \multicolumn{7}{|l|}{ Folate } \\
\hline Person-years & 292,275 & 290,461 & 290,196 & 292,381 & 291,364 & \\
\hline Cases, n & 107 & 83 & 79 & 89 & 69 & \\
\hline Model $1^{\mathrm{b}}$ & 1.00 (reference) & $0.80(0.60-1.07)$ & $0.81(0.60-1.08)$ & $0.88(0.66-1.17)$ & $0.67(0.49-0.92)$ & 0.03 \\
\hline Model $2^{c}$ & 1.00 (reference) & $1.02(0.76-1.37)$ & $1.10(0.82-1.49)$ & $1.24(0.93-1.67)$ & $1.00(0.72-1.37)$ & 0.71 \\
\hline Model $3^{d}$ & 1.00 (reference) & $1.01(0.74-1.36)$ & $1.10(0.80-1.51)$ & $1.27(0.92-1.75)$ & $1.07(0.74-1.55)$ & 0.50 \\
\hline \multicolumn{7}{|c|}{ 3-year exclusion analysis } \\
\hline Cases, n & 95 & 71 & 76 & 74 & 60 & \\
\hline Model $3^{d}$ & 1.00 (reference) & $0.97(0.70-1.34)$ & $1.19(0.85-1.65)$ & $1.18(0.84-1.68)$ & $1.04(0.70-1.54)$ & 0.65 \\
\hline \multicolumn{7}{|l|}{ Methionine } \\
\hline Person-years & 293,843 & 294,206 & 291,479 & 289,686 & 287,464 & \\
\hline Cases, n & 107 & 92 & 53 & 74 & 101 & \\
\hline Model $1^{\mathrm{b}}$ & 1.00 (reference) & $0.98(0.74-1.29)$ & $0.51(0.37-0.71)$ & $0.60(0.45-0.81)$ & $0.69(0.53-0.91)$ & 0.001 \\
\hline Model $2^{c}$ & 1.00 (reference) & $1.29(0.97-1.72)$ & $0.77(0.55-1.09)$ & $1.02(0.74-1.40)$ & $1.19(0.88-1.60)$ & 0.46 \\
\hline Model $3^{d}$ & 1.00 (reference) & $1.10(0.80-1.50)$ & $0.61(0.41-0.90)$ & $0.77(0.52-1.15)$ & $0.91(0.59-1.40)$ & 0.53 \\
\hline \multicolumn{7}{|c|}{ 3-year exclusion analysis } \\
\hline Cases, n & 96 & 81 & 48 & 66 & 85 & \\
\hline Model $3^{d}$ & 1.00 (reference) & $1.08(0.77-1.51)$ & $0.62(0.41-0.93)$ & $0.76(0.50-1.15)$ & $0.83(0.53-1.31)$ & 0.34 \\
\hline
\end{tabular}

${ }^{\mathrm{a}}$ Median values of vitamin $\mathrm{B} 12$, vitamin $\mathrm{B} 6$, folate, and methionine intakes in each quintile were used to test for a linear trend across .

${ }^{\mathrm{b}}$ Model 1 was adjusted for age, sex, and public health center area.

c Model 2 was adjusted for age, sex, public health center area, body mass index (quintiles), smoking (never, past, current; $\leq 19$ cigarettes/day, 20-29 cigarettes/day, or $\geq 30$ cigarettes/day), alcohol consumption (non-drinkers, $\leq 150 \mathrm{~g} /$ week, $150<$ alcohol $\leq 300 \mathrm{~g} /$ week, $300<$ alcohol $<450 \mathrm{~g} /$ week, or $\leq 450 \mathrm{~g} /$ week), family history of cancer, and physical activity in METs (quintiles).

${ }^{\mathrm{d}}$ Model 3 was model 2 that was mutually adjusted further for vitamin B12, vitamin B6, folate, and methionine 
Table 3 Hazard ratios (95\% confident intervals) of esophageal cancer according to quintiles of energy-adjusted dietary intakes of vitamin B12, vitamin B6, folate, and methionine- stratified analysis by alcohol consumption

\begin{tabular}{|c|c|c|c|c|c|c|c|}
\hline & Q1 & Q2 & Q3 & Q4 & Q5 & $p$-for trend ${ }^{a}$ & $p$-interaction \\
\hline Vitamin B12 & & & & & & & 0.18 \\
\hline \multicolumn{8}{|l|}{ Never drinker } \\
\hline Number at risk & 9,333 & 9,956 & 9,899 & 9,591 & 9,224 & & \\
\hline Person-years & 160,871 & 168,690 & 165,816 & 159,579 & 153,650 & & \\
\hline Case, $n$ & 7 & 11 & 19 & 26 & 23 & & \\
\hline Model $2^{b}$ & 1.00 (reference) & $1.45(0.56-3.75)$ & $2.31(0.96-5.53)$ & $3.27(1.39-7.66)$ & $2.82(1.18-6.74)$ & 0.009 & \\
\hline \multicolumn{8}{|c|}{ Alcohol intake 0-150 g/week } \\
\hline Number at risk & 2,554 & 3,142 & 3,425 & 3,697 & 3,380 & & \\
\hline Person-years & 44,250 & 53,644 & 57,610 & 61,874 & 56,835 & & \\
\hline Case, $n$ & 8 & 7 & 12 & 15 & 16 & & \\
\hline Model $2^{b}$ & 1.00 (reference) & $0.70(0.25-1.94)$ & $1.08(0.44-2.65)$ & $1.21(0.50-2.91)$ & $1.28(0.53-3.09)$ & 0.34 & \\
\hline \multicolumn{8}{|c|}{ Alcohol intake $>150 \mathrm{~g} /$ week } \\
\hline Number at risk & 5,161 & 3,878 & 3,665 & 3,721 & 4,372 & & \\
\hline Person-years & 85,509 & 63,418 & 60,921 & 61,546 & 71,490 & & \\
\hline Case, $n$ & 65 & 52 & 48 & 45 & 64 & & \\
\hline Model $2^{b}$ & 1.00 (reference) & $1.13(0.78-1.64)$ & $1.09(0.74-1.61)$ & $0.99(0.66-1.47)$ & $1.13(0.78-1.63)$ & 0.66 & \\
\hline Vitamin B6 & & & & & & & 0.89 \\
\hline \multicolumn{8}{|l|}{ Never drinker } \\
\hline Number at risk & 9,094 & 9,602 & 9,631 & 9,447 & 9,150 & & \\
\hline Person-years & 173,187 & 181,266 & 169,377 & 151,569 & 133,206 & & \\
\hline Case, $n$ & 10 & 16 & 20 & 21 & 19 & & \\
\hline Model $2^{b}$ & 1.00 (reference) & $1.37(0.62-3.03)$ & $1.58(0.73-3.39)$ & $1.69(0.78-3.64)$ & $1.53(0.69-3.37)$ & 0.31 & \\
\hline \multicolumn{8}{|c|}{ Alcohol intake 0-150 g/week } \\
\hline Number at risk & 2,627 & 3,108 & 3,494 & 3,622 & 3,347 & & \\
\hline Person-years & 45,054 & 53,227 & 59,136 & 61,086 & 55,710 & & \\
\hline Case, $n$ & 4 & 12 & 15 & 18 & 9 & & \\
\hline Model $2^{b}$ & 1.00 (reference) & $2.23(0.72-6.94)$ & $2.17(0.72-6.60)$ & $2.26(0.75-6.80)$ & $1.11(0.33-3.68)$ & 0.60 & \\
\hline \multicolumn{8}{|c|}{ Alcohol intake $>150 \mathrm{~g} /$ week } \\
\hline Number at risk & 4,478 & 3,205 & 3,385 & 4,236 & 5,493 & & \\
\hline Person-years & 73,024 & 53,030 & 56,065 & 70,334 & 90,430 & & \\
\hline Case, $n$ & 61 & 47 & 46 & 43 & 77 & & \\
\hline Model $2^{b}$ & 1.00 (reference) & $1.10(0.75-1.63)$ & $1.00(0.67-1.48)$ & $0.79(0.52-1.18)$ & $1.03(0.72-1.47)$ & 0.83 & \\
\hline Folate & & & & & & & 0.41 \\
\hline \multicolumn{8}{|l|}{ Never drinker } \\
\hline Number at risk & 7,560 & 9,277 & 9,946 & 10,401 & 10,819 & & \\
\hline Person-years & 129,722 & 156,106 & 166,190 & 175,262 & 181464 & & \\
\hline Case, $n$ & 13 & 12 & 19 & 27 & 15 & & \\
\hline Model $2^{b}$ & 1.00 (reference) & $0.74(0.33-1.69)$ & $1.19(0.57-2.50)$ & $1.43(0.71-2.92)$ & $0.76(0.35-1.69)$ & 0.80 & \\
\hline \multicolumn{8}{|c|}{ Alcohol intake 0-150 g/week } \\
\hline Number at risk & 2,662 & 3,339 & 3,454 & 3,397 & 3,346 & & \\
\hline Person-years & 45,040 & 56,139 & 58,275 & 57,803 & 56,956 & & \\
\hline Case, $\mathrm{n}$ & 10 & 9 & 13 & 20 & 6 & & \\
\hline Model $2^{b}$ & 1.00 (reference) & $0.65(0.26-1.61)$ & $0.93(0.40-2.14)$ & $1.40(0.64-3.05)$ & $0.40(0.14-1.12)$ & 0.30 & \\
\hline
\end{tabular}


Table 3 Hazard ratios (95\% confident intervals) of esophageal cancer according to quintiles of energy-adjusted dietary intakes of vitamin B12, vitamin B6, folate, and methionine- stratified analysis by alcohol consumption (Continued)

\begin{tabular}{|c|c|c|c|c|c|c|c|}
\hline & Q1 & Q2 & Q3 & Q4 & Q5 & $p$-for trend $d^{a}$ & $p$-interaction \\
\hline \multicolumn{8}{|c|}{ Alcohol intake $>150 \mathrm{~g} /$ week } \\
\hline Number at risk & 6,891 & 4,389 & 3,546 & 3,183 & 2,788 & & \\
\hline Person-years & 112,892 & 72,205 & 58,818 & 52,931 & 46,036 & & \\
\hline Case, $n$ & 84 & 58 & 44 & 42 & 46 & & \\
\hline Model $2^{b}$ & 1.00 (reference) & $1.12(0.79-1.57)$ & $1.06(0.73-1.54)$ & $1.05(0.72-1.55)$ & $1.30(0.89-1.89)$ & 0.25 & \\
\hline Methionine & & & & & & & 0.02 \\
\hline \multicolumn{8}{|l|}{ Never drinker } \\
\hline Number at risk & 8,214 & 10,251 & 10,241 & 9,929 & 9,368 & & \\
\hline Person-years & 141,928 & 174,731 & 172,663 & 164,817 & 154,605 & & \\
\hline Case, $n$ & 5 & 13 & 11 & 25 & 32 & & \\
\hline Model $2^{b}$ & 1.00 (reference) & $2.02(0.72-5.68)$ & $1.52(0.52-4.38)$ & $2.92(1.10-7.71)$ & $3.45(1.32-9.06)$ & 0.003 & \\
\hline \multicolumn{8}{|c|}{ Alcohol intake 0-150 g/week } \\
\hline Number at risk & 2,031 & 2,743 & 3,445 & 4,001 & 3,978 & & \\
\hline Person-years & 34,893 & 47,437 & 57,881 & 67,544 & 66,457 & & \\
\hline Case, $n$ & 4 & 10 & 14 & 16 & 14 & & \\
\hline Model $2^{b}$ & 1.00 (reference) & $1.68(0.53-5.37)$ & $1.54(0.50-4.74)$ & $1.37(0.45-4.14)$ & $1.06(0.34-3.27)$ & 0.52 & \\
\hline \multicolumn{8}{|c|}{ Alcohol intake $>150 \mathrm{~g} /$ week } \\
\hline Number at risk & 6,852 & 3,956 & 3,309 & 3,067 & 3,613 & & \\
\hline Person-years & 112,220 & 65,083 & 54,758 & 51,056 & 59,764 & & \\
\hline Case, $n$ & 98 & 65 & 28 & 32 & 51 & & \\
\hline Model $2^{b}$ & 1.00 (reference) & $1.23(0.89-1.70)$ & $0.64(0.42-0.99)$ & $0.80(0.53-1.22)$ & $1.03(0.72-1.48)$ & 0.68 & \\
\hline
\end{tabular}

Abbreviations: $\mathrm{HR}$, hazard ratio; $95 \% \mathrm{Cl}, 95 \%$ confidence interval.

${ }^{a}$ Median values of vitamin B12, vitamin B6, folate, and methionine in each quintile were used to test for a linear trend across quintiles.

${ }^{b}$ Model 2 was adjusted for age, sex, public health center area, body mass index (quintiles), smoking (never, past, current; $\leq 19$ cigarettes/day, $20-29$ cigarettes/ day, or $\geq 30$ cigarettes/day), alcohol consumption (non-drinkers, $\leq 150 \mathrm{~g} /$ week, $150<$ alcohol $\leq 300 \mathrm{~g} /$ week, $300<$ alcohol<450 g/week, or $\geq 450 \mathrm{~g} /$ week) for the everdrinkers' group, family history of cancer, and physical activity in METs (quintiles)

drinkers but not among drinkers, either low or high intake alcohol drinkers. The other investigated nutrients, vitamin $\mathrm{B} 6$ and folate, were not associated with the risk of EC.

\section{Dietary intake of vitamin B12}

The positive association between the dietary intake of vitamin $\mathrm{B} 12$ and the risk of $\mathrm{EC}$ was consistent with previous studies conducted in the USA and Europe $[15,16]$. One of the studies that found a positive association between vitamin B12 intake and EC risk was a case-control study in the USA, which included 687 controls, 282 cases of esophageal adenocarcinoma, and 206 cases of esophageal squamous cell carcinoma. They found that the odds ratio (OR) $(95 \% \mathrm{CI})$ of dietary vitamin B12 intake (comparing $\geq 75^{\text {th }}$ percentile of intake to $25^{\text {th }}$ percentile of intake) in esophageal adenocarcinoma versus control was $1.39(1.10-1.76)$ and that in esophageal squamous cell carcinoma versus control was 1.51 (1.152.00) [15]. Another study that found a positive association between dietary intake of vitamin B12 and the risk of EC was a case-control study within the FINBAR study in Ireland that included 227 cases of esophageal adenocarcinoma and 260 controls. In that study, dietary vitamin B12 intake was positively associated with esophageal adenocarcinoma risk; the multivariable OR $(95 \% \mathrm{CI})$ in the highest $(\geq 9.7 \mu \mathrm{g} /$ day $)$ versus lowest $(\leq 4$ $\mu \mathrm{g} /$ day) quartiles of dietary vitamin B12 intake was 3.87 (2.22-6.73; $p$-trend was $\leq 0.01)$ [16]. The mechanism of the positive association between the dietary intake of vitamin $\mathrm{B} 12$ and risk of $\mathrm{EC}$ is still unclear. In the previous studies, the observed positive association were mainly attributed to the fact that the primary sources of vitamin B12 were animal origin foods, such as meat or fish [16, 29]. Patients with esophageal adenocarcinoma versus controls in the FINBAR study in Europe were more likely to consume higher amounts of fresh red meat; the multivariate OR (95\% CI) was 3.15 (1.38-7.20) [30]. In Japan, the main dietary sources of vitamin B12 have been shown to be fish and shellfish, followed by meat [31]. We analyzed the data of our study to examine the association of fish and shellfish, and total meat intake with EC risk; however, we did not find any significant association for either food. We also examined the 
association between the intake of dairy products and eggs, other food sources of vitamin B12, and the risk of $\mathrm{EC}$, and we found no association between them (not shown in table). Furthermore, there was no significant change in the association between vitamin B12 and the risk of EC in the fully-adjusted model that included fish and shellfish, total meat, eggs, and dairy products (HR and 95\%CI 1.73[1.11-2.68], $p$-trend=0.01) (not shown in table). A molecular study found that aberrant DNA methylation might be involved in the pathogenesis of esophageal squamous cell carcinoma [32]. In addition, a meta-analysis study showed that one-carbon metabolism nutrients were associated with aberrant DNA methylation [33]. Despite that, the complex nature of the overall dietary patterns and disease risk association suggests that the positive association between high vitamin B12 intake and the risk of EC might reflect that vitamin B12 is a surrogate of pro-carcinogenic diet or vitamin B12 per se may increase the cancer risk; however, further research need to be conducted to investigate this hypothesis.

One carbon-metabolism nutrients act interactively to affect health and cancer pathogenesis [34]. Therefore, to control the interactions between $B$ vitamins in this study, we conducted the analysis with a mutually adjusted model that included all of the B vitamins and methionine simultaneously (Model 3). We observed that the positive association between vitamin $\mathrm{B} 12$ and the risk of EC became stronger after controlling for other studied B vitamins and methionine.

The positive association between vitamin B12 intake and EC risk was only found among participants who were not alcohol-drinker. However, $p$-interaction with alcohol intake did not reach statistical significance ( $p$-interaction $=0.18$ ). A similar finding was also observed in men; however, we could not conduct the stratified analysis based on alcohol intake in women due to the small number of cases. Biologically, alcohol could cause malabsorption of vitamin B12 and decrease its bioavailability [21, 35]. A randomized controlled trial showed a 5\% decrease in blood vitamin B12 levels in postmenopausal women who had been given $15 \mathrm{~g}$ and $30 \mathrm{~g}$ alcohol for 8 weeks compared with subjects who had not been given alcohol [21]. Consistent with this finding, another randomizedcontrolled trial found a $6 \%$ decrease in plasma vitamin B12 levels in male volunteers after a 2-week red wine and vodka intervention [35]. The positive association between higher dietary intake of vitamin B12 and EC risk that was only found among alcohol never-drinkers may be because there is no reduction in vitamin B12 bioavailability in the bodies of these individuals. The cancer-promoting effect of vitamin B12 may be weaker than that of alcohol, a strong risk factor for EC [36, 37]. Therefore, this could have also been the reason for not detecting the effect of vitamin B12 on EC risk in low and high alcohol drinkers.

\section{Dietary intake of methionine}

There was no association found between dietary methionine intake and EC risk. In the stratified analysis based on alcohol consumption, we found a significant positive association between dietary methionine intake and EC risk among never-drinkers. This positive association was similar to that of vitamin B12. In fact, there was a high Pearson correlation coefficient between dietary intakes of methionine and vitamin B12 ( $\mathrm{r}=0.74 ; p<0.0001)$.

Biologically, vitamin B12 is an essential substrate of the methionine cycle in the OCM pathway [38]. An animal study showed a decrease in methionine uptake in the intestines of rats after 40 days of alcohol intake [39]. Thus, an effect of methionine on the risk of EC was expected among never-drinkers.

\section{Dietary intake of folate and vitamin B6}

There was no association between dietary intake of folate and the risk of EC. Our finding is supported by a cohort study from Uruguay, which found that the multivariable $\mathrm{HR}(95 \% \mathrm{CI})$ of $\mathrm{EC}$ in the highest versus lowest quartiles of dietary folate intake was $1.15(0.83-1.59 ; p=$ 0.34) [40]. On the other hand, several other studies reported an inverse association between dietary intake of folate and risk of EC, even after multivariable adjustment.[14-19, 41] The biological justification for this discrepancy is currently lacking; however, in the studies that found an inverse association between dietary folate intake and EC risk, the median folate intake in the highest quintile or quartile was lower than that in our study: $638 \mu \mathrm{g} /$ day in our study versus $379 \mu \mathrm{g} /$ day and $275 \mu \mathrm{g} /$ day in the studies by Ibiebele et al. and Aune et al., respectively [14, 18]. The NIH-AARP Diet and Health Study from the USA, which had a similar high intake of folate (median intake values of $288 \mathrm{mcg} /$ day in Q1 up to $566 \mathrm{mcg} /$ day in Q5) and used Q3 (405 mcg/day) as the reference category, found an increased risk of esophageal squamous cell carcinoma with lower intake of folate: HR (95\% CI) for Q1 versus Q3 was 1.91 (1.17-3.10); however, no reduced risk was found with higher folate intake: HR (95\% CI) for Q5 versus Q3 was 1.07 (0.591.94) [19]. Although the FFQ was not able to measure absolute nutrient intake and thus, did not allow its comparison with the RDA, it is worth noting that the dietary folate intake in Q5 in our study was higher than that in the Japanese RDA, even though Japan does not have mandatory folic acid food fortification policy [42]. Our study may be in line with the NIH-AARP Diet and Health Study that found no association between folate intake and EC risk among those with a higher dietary folate intake [19].

There was no association between the dietary intake of vitamin B6 and risk of EC, which was consistent with the results of a cohort study from the USA [19]. 
However, a case-control study from Ireland by Sharp et al. reported an inverse association between vitamin B6 intake and EC risk; the OR (95\% CI) in the highest versus lowest quartiles of vitamin B6 intake was 0.37 $(0.22-0.63 ; p$-trend was <0.01) [16]. This discrepancy may be due to the different adjustment factors that were used. Compared with their study that did not include smoking status, our study included smoking status in the analyses [16]. It is worth noting that Sharp et al. found inverse associations of dietary intake of folate and vitamin B6 only with the risk of esophageal adenocarcinoma as they did not have cases of esophageal squamous cell carcinoma [16]. In our study, the dominant histological EC subtype was esophageal squamous cell carcinoma. An Australian case-control study that included 364 esophageal adenocarcinoma and 306 esophageal squamous cell carcinoma cases reported inverse associations between folate and vitamin B6 intakes and esophageal adenocarcinoma risk. For esophageal adenocarcinoma, the multivariable HRs (95\% CIs) in the highest versus lowest quartiles of intakes were $0.72(0.53-0.98 ; p$-trend= $0.01)$ and $0.53(0.39-0.74 ; p$-trend $=0.002)$ for folate and vitamin B6, respectively; however, no associations with the risk of esophageal squamous cell carcinoma were found; HRs were $0.78(0.51-1.19 ; p$-trend $=0.06)$ and $0.66(0.42-$ 1.05 ; $p$-trend $=0.08)$ for dietary intakes of folate and vitamin B6, respectively [18].

In our study, the dominant histological EC subtype was squamous cell carcinoma, and it mostly occurred in men; this was consistent with previous findings in Japan that esophageal squamous cell carcinoma accounted for $89.5 \%$ of all EC cases, and $84.9 \%$ of these esophageal squamous cell carcinoma cases occurred in men [43]. Due to the small number of esophageal adenocarcinoma cases, we could not conduct a stratified analysis based on histological subtype. However, when we limited the analysis to esophageal squamous cell carcinoma, there was an increased risk of esophageal squamous cell carcinoma in men with higher dietary intakes of vitamin B12 (data not shown).

\section{Strengths and limitations}

This was the first large prospective cohort study to investigate the association of $\mathrm{B}$ vitamins and methionine intake with EC risk in Japan. Our study had several strengths, including a long follow-up time, validated dietary intakes, and a large sample size, which allowed the stratified analyses based on alcohol intake to detect the possible effect modification by alcohol consumption.

Nevertheless, this study also had some limitations. First of all, the blood biomarkers of vitamin B12 status were not assessed. According to previous research from the JPHC study, the blood measurement of vitamin B12 status was only conducted in less than $1 \%$ of the JPHC population. Furthermore, the observed correlation coefficients between dietary intakes of vitamin B12, vitamin B6, and folate with the blood concentrations of these vitamins were relatively low, especially for vitamin B12; Spearman correlations value were $0.06,0.23$, and 0.23 , respectively [44]. Dietary intake of vitamin B12 is a relatively poor reflection of vitamin B12 status because the bioavailability of vitamin B12 may be affected by individual variance in vitamin B12 absorption [45]. For example, malabsorption of vitamin B12 could be caused by chronic gastric inflammation caused by atrophic gastritis, inflammatory bowel disease, or prolonged use of drugs that affect the $\mathrm{pH}$ of the stomach such as protonpump inhibitors and $\mathrm{H} 2$-receptor antagonists, and metformin by affecting the vitamin B12 receptor [46-49].

Second, the most dominant histological EC subtype was squamous cell carcinoma, and there were only a few esophageal adenocarcinoma cases $(n=17)$; thus, we could not evaluate the associations of $B$ vitamins and methionine intake with adenocarcinoma EC subtype risk specifically.

Third, we lacked information on the use of vitamin B supplementation; however, the use of vitamin supplements during the time of the baseline survey (1990s) was not common in Japan. Finally, information about gastroesophageal reflux disease or Barrett's esophagus as strong risk factors for $\mathrm{EC}$ was unavailable.

\section{Conclusions}

Our study showed that a higher dietary intake of vitamin B12 was associated with an increased risk of EC. In the stratified analysis by alcohol intake status, the positive associations of vitamin B12 and methionine intake with the risk of EC were found among never-drinkers. On the other hand, there was no significant association between vitamin $\mathrm{B} 12$ and methionine intake and the risk of EC among both low-level (0-150 g/week) and high-level (>150 g/week) alcohol drinkers. Future research is warranted on the association between vitamin $\mathrm{B} 12$ and the risk of EC in other populations.

\section{Abbreviations}

BMI: Body mass index; Cl: Confidence interval; EC: Esophageal cancer; FFQ: Food Frequency Questionnaire; HR: Hazard ratio; JPHC: Japan Public Health Center-Based Prospective Study; MET: Metabolic equivalent of task; OCM: One-carbon metabolism; OR: Odds ratio; PHC: Public health center; RDA: Recommended Dietary Allowance

\section{Supplementary Information}

The online version contains supplementary material available at https://doi. org/10.1186/s12885-021-08721-8.

\section{Additional file 1.}

\section{Acknowledgments}

JPHC members are listed on the following website (as of September 2019): https://epi.ncc.go.jp/en/jphc/781/8390.html. We are indebted to the Aomori, 
Akita, Iwate, Ibaraki, Niigata, Osaka, Kochi, Nagasaki, and Okinawa Cancer Registries for providing their incidence data.

\section{Reporting guidelines}

Our manuscript was written in accordance with STROBE guidelines, a completed checklist has been provided.

\section{Authors' contributions}

PK, TS, and ESE designed the study. NS, MI, and ST performed the follow-up survey and collected the data. PK performed the statistical analysis of the data and wrote the manuscript. PK, TS, HI, ESE, LZ were involved in the interpretation of the data. All authors revised the manuscript critically for important intellectual content. All authors read and approved the final manuscript.

\section{Funding}

This study was supported by the National Cancer Centre Research and Development Fund (since 2011), and a Grant in Aid for Cancer research from the Ministry of Health, Labour and Welfare of Japan (from 1989 to 2010). The funders played no role in design, interpretation or publication of study findings.

\section{Availability of data and materials}

The datasets generated and/or analysed during the current study are not publicly available due to participant privacy, according to ethical guidelines in Japan. Additionally, the informed consent we obtained does not include a provision for publicly sharing data. The data are available from the JPHC Study Group at https://epi.ncc.go.jp/en/jphc/805/8155.html or the Office of The JPHC Study Group at jphcadmin@ml.res.ncc.go.jp on reasonable request.

\section{Declarations}

\section{Ethics approval and consent to participate}

The study was conducted in compliance with the provisions of the Declaration of Helsinki.

The study protocol was approved by the Institutional Review Board of the National Cancer Center, Tokyo, Japan (approval number: 2001-013, 14-038). The present study protocol was approved by the Ethical Review Board of Osaka University, Osaka, Japan (approval number: 14020-9). All participants were informed of the objectives of the study and that completion of the survey questionnaire was regarded as providing informed consent.

\section{Consent for publication}

Not applicable.

\section{Competing interests}

The authors declare that there are no competing interests.

\section{Author details}

${ }^{1}$ Department of Social and Environmental Medicine, Graduate School of Medicine, Osaka University, 2-2 Yamadaoka, Suita, Osaka 565-0871, Japan. ${ }^{2}$ Department of Internal Medicine, Faculty of Medicine, University of Muhammadiyah, Jakarta, Indonesia. ${ }^{3}$ Public Health, Department of Social Medicine, Osaka University Graduate School of Medicine, Suita, Osaka 565-0871, Japan. ${ }^{4}$ Department of Public Health and Preventive Medicine, Faculty of Medicine, Minia University, Minya, Egypt. ${ }^{5}$ Epidemiology and Prevention Group, Center for Public Health Sciences, National Cancer Center, Chuo-ku Tokyo 104-0045, Japan.

\section{Received: 8 April 2021 Accepted: 18 August 2021}

\section{Published online: 01 September 2021}

\section{References}

1. Bray F, et al. Global cancer statistics 2018: GLOBOCAN estimates of incidence and mortality worldwide for 36 cancers in 185 countries. CA. Cancer J. Clin. 2018;68:394-424.

2. Wang QL, Xie SH, Wahlin K, Lagergren J. Global time trends in the incidence of esophageal squamous cell carcinoma. Clin. Epidemiol. 2018;10:717-28.

3. World Cancer Research Fund \& American Institute for Cancer Research. Diet, nutrition, physical activity and oesophageal cancer. Continous Update Project https://www.wcrf.org/sites/default/files/Oesophageal-cancerreport.pdf (2018).
4. Bravi F, et al. Dietary patterns and the risk of esophageal cancer. Ann. Oncol. 2012;23:765-70.

5. Gilsing AMJ, et al. Serum concentrations of vitamin B12 and folate in British male omnivores, vegetarians and vegans: Results from a cross-sectional analysis of the EPIC-Oxford cohort study. Eur. J. Clin. Nutr. 2010;64:933-9.

6. Zhu HC, et al. Meat consumption is associated with esophageal cancer risk in a meat- and cancer-histological-type dependent manner. Dig. Dis. Sci. 2014;59:664-73.

7. Coppedè $F$, et al. Association of polymorphisms in genes involved in one-carbon metabolism with MTHFR methylation levels. Int. J. Mol. Sci. 2019;20:1-11.

8. Mandaviya PR, et al. Association of dietary folate and Vitamin B-12 intake with genome-wide DNA methylation in blood: A large-scale epigenome-wide association analysis in 5841 individuals. Am. J. Clin. Nutr. 2019;110:437-50.

9. Newman AC, Maddocks ODK. One-carbon metabolism in cancer. Br. J. Cancer. 2017;116:1499-504.

10. Banjari I, Kožić S. Dietary intake of vitamin B 12 in relation to diet and lifestyle characteristics in a population at high risk for colorectal cancer. Cent. Eur. J. Public Health. 2018;26:253-9.

11. Hirsch $\mathrm{S}$, et al. Colon cancer in Chile before and after the start of the flour fortification program with folic acid. Eur. J. Gastroenterol. Hepatol. 2009;21: 346-9.

12. Gong Z, et al. Associations of dietary folate, Vitamins B6 and B12 and methionine intake with risk of breast cancer among African American and European American women. Int. J. Cancer. 2014;134:1422-35.

13. Takata $Y$, et al. Food intake of folate, folic acid and other $B$ vitamins with lung cancer risk in a low-income population in the Southeastern United States. Eur. J. Nutr. 2020:59:671-83.

14. Aune D, et al. Dietary folate intake and the risk of 11 types of cancer: A case-control study in Uruguay. Ann. Oncol. 2011;22:444-51.

15. Mayne ST, et al. Nutrient Intake and Risk of Subtypes of Esophageal and Gastric Cancer. Cancer Epidemiol. Biomarkers Prev. 2001;10:1055-62.

16. Sharp L, Carsin A-E, Cantwell MM, Anderson LA, Murray LJ. Intakes of dietary folate and Other B Vitamins Are Associated with Risks of Esophageal Adenocarcinoma, Barrett's Esophagus, and Reflux Esophagitis. J. Nutr. 2013; 143(12):1966-73.

17. Galeone C, et al. Folate intake and squamous-cell carcinoma of the oesophagus in Italian and Swiss men. Ann. Oncol. 2006;17:521-5.

18. Ibiebele TI, et al. High Intake of Folate from Food Sources Is Associated with Reduced Risk of Esophageal Cancer in an Australian Population. J. Nutr. 2011;141:274-83.

19. Xiao $\mathrm{Q}$, et al. Intakes of folate, methionine, vitamin B6, and vitamin B12 with risk of esophageal and gastric cancer in a large cohort study. Br. J. Cancer. 2014;110:1328-33.

20. Halsted CH, Villanueva JA, Devlin AM, Chandler CJ. Metabolic Interactions of Alcohol and Folate. J. Nutr. 2002;132:2367S-72S.

21. Laufer EM, et al. Effects of moderate alcohol consumption on folate and vitamin B12 status in postmenopausal women. Eur. J. Clin. Nutr. 2004;58:1518-24.

22. Tsugane S, Sawada N. The JPHC study: Design and some findings on the typical Japanese diet. Jpn. J. Clin. Oncol. 2014;44:777-82.

23. Science and Technology Agency. Standard Tables of Food Composition in Japan. (Printing Bureau of the Ministry of Finance, 2015).

24. Willett WC, Howe R. Adjustmentfor total energyintake in epidemiologic studies. Am J Clin Nutr. 1997:65:1220S-8S.

25. Okada $C_{\text {, }}$ et al. Validity and reliability of a self-administered food frequency questionnaire for the JPHC study: The assessment of amino acid intake. J. Epidemiol. 2017;27:242-7.

26. Ishihara J, et al. Impact of the revision of a nutrient database on the validity of a self-administered food frequency questionnaire (FFQ). J. Epidemiol. 2006;16:107-16

27. World Health Organization et al. ICDO — International Classification of Diseases for Oncology. World Health Organization (2000). doi:10.1016/s00313025(16)36112-8.

28. Li Y, et al. Alcohol Consumption and Risk of Gastric Cancer: The Japan Collaborative Cohort Study. J Epidemiol. 2019;31(1):30-6. https://doi.org/1 0.2188/jea.je20190304.

29. Ma J-L, et al. Dietary vitamin B intake and the risk of esophageal cancer: a meta-analysis. Cancer Manag. Res. 2018;10:5395-410.

30. O'Doherty MG, Cantwell MM, Murray $\amalg$, Anderson LA, Abnet CC. Dietary fat and meat intakes and risk of reflux esophagitis Barrett's esophagus and esophageal adenocarcinoma. Int. J. Cancer. 2011;129:1493-502. 
31. Yoshino K, et al. Trends in Dietary Intake of Folate, Vitamins B6, and B12 among Japanese Adults in Two rural communities from 1974 through 2001. J. Epidemiol. 2006;15:29-37.

32. Chen C, et al. Genome-wide profiling of DNA methylation and gene expression in esophageal squamous cell carcinoma. Oncotarget. 2016;7: 4507-21.

33. Amenyah $S D$, et al. Influence of nutrients involved in one-carbon metabolism on DNA methylation in adults-a systematic review and metaanalysis. Nutr. Rev. 2020;0:1-20.

34. Locasale JW. Serine, glycine and one-carbon units: Cancer metabolism in full circle. Nat. Rev. Cancer. 2013;13:572-83.

35. Gibson A, et al. Alcohol increases homocysteine and reduces B vitamin concentration in healthy male volunteers - A randomized, crossover intervention study. Q J Med. 2008;101:881-7.

36. Kimm H, Kim S, Jee SH. The independent effects of cigarette smoking alcohol consumption, and serum aspartate aminotransferase on the alanine aminotransferase ratio in Korean men for the risk for esophageal cancer. Yonsei Med. J. 2010;51:310-7.

37. Kumagai $\mathrm{N}$, et al. Heavy alcohol intake is a risk factor for esophageal squamous cell carcinoma among middle-aged men: A case-control and simulation study. Mol. Clin. Oncol. 2013;1:811-6.

38. Rush EC, Katre P, Yajnik CS. Vitamin B12: One carbon metabolism, fetal growth and programming for chronic disease. Eur. J. Clin. Nutr. 2014;68:2-7.

39. Polache A, Martín-Algarra RV, Guerri C. Effects of chronic alcohol consumption on enzyme activities and active methionine absorption in the small intestine of pregnant rats. Alcohol. Clin. Exp. Res. 1996;20:1237-42.

40. De Stefani $\mathrm{E}$, et al. Nutrient patterns and risk of squamous cell carcinoma of the esophagus: A factor analysis in Uruquay. Nutr. Cancer. 2006;56:149-57.

41. Jessri M, Rashidkhani B, Hajizadeh B, Jessri M, Gotay C. Macronutrients, vitamins and minerals intake and risk of esophageal squamous cell carcinoma: A case-control study in Iran. Nutr. J. 2011;10:137.

42. Food Fortification Initiative. Japan- Country Profile. 4-6 (2021).

43. Tachimori Y, et al. Comprehensive registry of esophageal cancer in Japan, 2012. Esophagus. 2019;16:221-45.

44. Iso $\mathrm{H}$, et al. Validity of the self-administered food frequency questionnaire used in the 5-year follow-up survey for the JPHC Study to assess folate, vitamin B6 and B12 intake: Comparison with dietary records and blood level. J. Epidemiol. 2003;13:98-101.

45. Obeid R, et al. Vitamin B12 intake from animal foods, biomarkers, and health aspects. Front. Nutr. 2019;6:93. https://doi.org/10.3389/fnut.2019.00093.

46. Aroda VR, et al. Long-term metformin use and vitamin B12 deficiency in the diabetes prevention program outcomes study. J. Clin. Endocrinol. Metab. 2016;101:1754-61.

47. Miller JW. Proton Pump Inhibitors, H2-Receptor Antagonists, Metformin, and Vitamin B-12 Deficiency: Clinical Implications. Adv. Nutr. 2018;9:511S-8S.

48. Cavalcoli F, Zilli A, Conte D, Massironi S. Micronutrient deficiencies in patients with chronic atrophic autoimmune gastritis: A review. World J. Gastroenterol. 2017:23:563-72.

49. Park YE, et al. Incidence and risk factors of micronutrient deficiency in patients with IBD and intestinal Behçet's disease: folate, vitamin B12, 25-OHvitamin D, and ferritin. BMC Gastroenterol. 2021;21:1-9.

\section{Publisher's Note}

Springer Nature remains neutral with regard to jurisdictional claims in published maps and institutional affiliations.

Ready to submit your research? Choose BMC and benefit from:

- fast, convenient online submission

- thorough peer review by experienced researchers in your field

- rapid publication on acceptance

- support for research data, including large and complex data types

- gold Open Access which fosters wider collaboration and increased citations

- maximum visibility for your research: over $100 \mathrm{M}$ website views per year

At $\mathrm{BMC}$, research is always in progress.

Learn more biomedcentral.com/submissions 